\title{
USING THE COMBINED LADM-IndoorGML MODEL TO SUPPORT BUIILDING EVACUATION
}

\author{
A. Alattas ${ }^{1,2, *}$, P. van Oosterom ${ }^{2}$, S. Zlatanova ${ }^{3}$, D. Hoeneveld ${ }^{4}$, E. Verbree $^{2}$ \\ ${ }^{1}$ Faculty of Environmental Design, Geomatics department, King Abdulaziz University, Jeddah, Saudi Arabia - \\ Arch.alattas@gmail.com \\ ${ }^{2}$ Faculty of Architecture and the Built Environment, Delft University of Technology, Julianalaan 134, 2628 BL Delft, The \\ Netherlands - \{A.F.M.Alattas, P.J.M.vanOosterom, E.Verbree $\} @$ tudelft.nl \\ ${ }^{3}$ Faculty of Built Environment, UNSW, Red Centre Building, NSW 2052, Sydney, Australia - s.zlatanova@unsw.edu.au \\ ${ }^{4}$ Faculty of Technology, Policy and Management, Delft University of Technology, Jaffalaan 5, 2628 BX Delft, The Netherlands - \\ D.Hoeneveld@tudelft.nl
}

Commission IV, WG IV/5

KEY WORDS: Indoor modelling, UML, Access, Security, LADM, IndoorGML

\begin{abstract}
:
During an incident, many people that are located in indoor environments require to follow emergency evacuation procedures. The 'emergency evacuation' term has been defined as 'a critical movement of people from a dangerous area due to the risk or an incident of a tragic event' (Bonabeau, 2002). An emergency evacuation could be needed in a life or death situation, regardless if it begins with a natural non-intended incident or a terrorist attack. Many researchers have studied the behaviour of the people during the evacuation because of several incidents with panic attacks that have led to injuries including death of people being crushed or trampled down by others. In crisis situation, the perception of the indoor environment, which differs from person to person, play a critical role in the evacuation. Also, the access rights of the indoor spaces are different from those rights (and restrictions) during normal times. They may positively impact the movements of the people during the evacuation by providing suggestions for shorter/ better route. This paper addresses the impact of the access rights of the indoor spaces during an emergency evacuation. We employ the conceptual model of LADM-IndoorGML that defines the accessibility of the indoor spaces based on the rights, restrictions, and responsibilities of the user of the indoor space. The access rights of the indoor spaces are affected by the crisis event and this needs to be modelled explicitly (and before crisis situation). Actually, the rights/ restrictions persons have on spaces is time dependent: normal operation hours, outside normal operation hours (e.g. during night time in case of a University building) or during crisis times. These actual/ valid rights and restrictions affect the movement / accessibility of the users to reach the nearest emergency exits or the safe zone. For this reason, different scenarios have to be developed to study the impact of the accessibilities for different types of users. In this paper we will present the 3D model of an educational building that was built for the purpose of evacuation study. The 3D model is supported by real data for all spaces from the facility management department such as information on departments, sections, groups of users (visitors, employees, and students), and public/private spaces, etc. and a real evacuation exercise. We consider it extremely important to develop our information model based on international standards (LADM/ISO 19152, OGC IndoorGML, ISO 19141, ISO 19107) as we expect that this information will be part of the future 'building infrastructure' and applications all over the world can understand and use this data when entering or leaving a certain building both during normal and crisis situations. Different types of applications are anticipated to be based on this information model; e.g. mobile indoor routing app (for normal building users and Emergency Response Team members), crisis evacuation desktop application for command centre, etc.
\end{abstract}

\section{INTRODUCTION}

During an incident in the indoor environment, an efficient evacuation is required to: help and protect a large number of people by evacuating them from the incident zone and the surrounding areas to a safe area, which is usually outside the building. The indoor environment is a sophisticated space during the normal situation, and that complexity increases during an incident. The users of the indoor environment must find the safest route from their location to the emergency exit. For this reason, several researchers have concentrated on the modeling of the network and the indoor wayfinding (Xiong et al., 2017). Many emergency evacuations models have been developed on different computational rules and to represent effective approaches for the designers to estimate the evacuation safety during the integration with GIS-based spatial models such as Firescap, Exoudus, Simulex, Esm, and Mascm (Tang and Ren, 2012). Additionally, their study proposed a spatial simulation model that merge behaviours, fire simulation, and building geometry to examine the spatial features and support the evacuation decision makers to a certain level.

A 3D indoor-outdoor spatial model has been proposed by (Tashakkori et al. 2015). They have introduced the required information for finding the route during the emergency incident response, and they included information about the building and the network between the floors. Furthermore, they discussed the awareness of the situation that is needed for managing the emergency and for the decision that will be performed for a successful emergency respond according to the 3D network

\footnotetext{
* Corresponding author
} 
navigation and blockage analysis. (Lujak et al. 2017) have introduced a distributed recommendation route for a situationaware evacuation guidance in a building.

Additional, several pedestrian simulation models have been developed for different disciplines such as computer graphics, robotics, and evacuation dynamics. These models could be categorized into two groups: macroscopic and microscopic models (Pelechano et al., 2008). The macroscopic model contains a route choice model that describes the pedestrian wayfinding based on the idea of utility and that by choosing their destinations based on the utility of their trips such as the comfort and the travel time (Hoogendoorn et al., 2005). The lack of information about the internal connectivity of the indoor spaces usually slow down the evacuation, and in this case, some of the users will not know the alternative route to the emergency exit. Users of the indoor spaces usually take the same route that they use during the normal situation and ignore the emergency route or exit that should be taken during the incident (R. Sime, 1984).

However, those researchers have not addressed the necessary information to identify the access rights for the users during the evacuation and how that will affect the evacuation routes for the users of the indoor environment during the incident. There are two types of spaces for the users based on their rights: (1) accessible spaces (2) non-accessible spaces during the regular daily activity inside the building. Therefore, it is essential to examine the impact of the access right for the users during the incident and store this information which can help to improve generating better routes that could be shorter and safer.

This paper addresses the impact of the access rights of the indoor spaces during an emergency evacuation by employing the conceptual model of LADM-IndoorGML that was defined by (Alattas et al. 2017) to determine the accessibility of the indoor spaces during an incident based on the rights, restrictions, and responsibilities. The conceptual model of LADM-IndoorGML determines the accessibility rights over time to offer effective navigation. The LADM-IndoorGML conceptual model determines the relationship between the spaces of the indoor environments and the users by applying the LADM to assign the rights, restrictions, and responsibilities for each space to define the accessible spaces for each type of users. IndoorGML is an Open Geospatial Consortium (OGC) describes an indoor navigation framework that clarifies the indoor space and the Geographic Markup Language (GML) syntax for the encoding geoinformation (Zlatanova et al. 2016). The Land Administration Domain Model (LADM) is an ISO standard (19152:2012) that focus on rights, restrictions, and responsibilities (RRR) that affect the lands and space (Lemmen et al. 2015).

Currently, navigation during the incident has been studied without any consideration of the impact of the access rights for the users; therefore, this paper assesses the conceptual model of
LADM-IndoorGML to define the accessibility rights during an incident. A real evacuation exercise for an educational building at the Technical University of Delft is considered. Two scenarios have been prepared for the evacuation and Wi-Fi trajectory data have been collected to study and compare the movement of the users during the evacuation. A 3D model has been created for the building and several types of information for each space such as space type, area, space function, level etc. have been defined.

This paper is organized as follows: in Section 2, we discuss the LADM-IndoorGML Conceptual Model During Crisis, section 3 represents the evacuation exercise scenarios, and section 4 describes the result of the evacuation scenarios. The paper ends with conclusions in section 5 .

\section{LADM-INDOORGML CONCEPTUAL MODEL DURING CRISIS}

The conceptual model of LADM-IndoorGML defines the access rights for the users of the indoor environment as shown in Figure 1. Each space of the indoor environments has a different relationship with the users, and by determining these relationships, LADM could define access right for each type of users. Therefore, there are two kinds of spaces defined by LADM: common right spaces and private right spaces and based on the user rights, the indoor space can be subdivided with respect to each individual user or a group of users. The core model of LADM consists of three packages and one subpackage: Party Package, Administrative Package, Spatial Package and the Surveying and representation sub-package (ISO19152:2012).

The Party package responsible for the representation of the user information, and it contains LA_Party class, LA_GroupParty, and LA_PartyMember as an optional association class. The Party package has been developed to cover more information about the users of the indoor Environment for better representation and that by including three more classes (Alattas et al. 2017). The refinement of the Party package has added two subclasses to the LA_GroupParty class to represent particular groups of users based on the type of the indoor environment: (1) LA_GroupStudent and LA_GroupEmployee. The additional two classes of the LA_GroupParty will inherit the attributes of LA_Party and LA_GroupParty classes based on the type of association that connect the classes. The Current LA_GroupParty has been extended to have a new subclass to represent the Emergency Response Team (ERT). The new subclass has a LA_GroupEmergencyResponseTeamType attribute to represent several types of Emergency Response Teams such as Emergency Medical Technician, Firefighters, Police, and ERT as shown in Figure 3. The aim of the new subclass is to define the access rights during an incident for the Emergency Response Team users. 


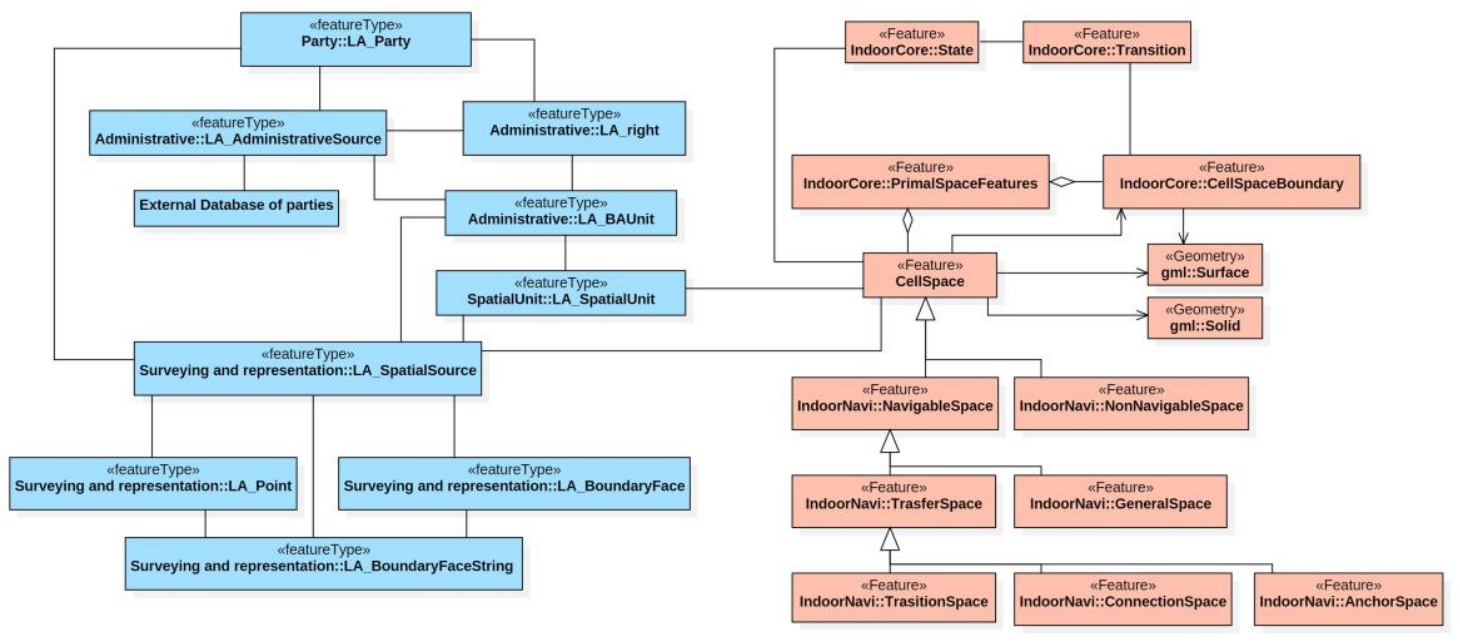

Figure 1. The conceptual model of LADM (blue) and IndoorGML (red) (Alattas et al. 2017)
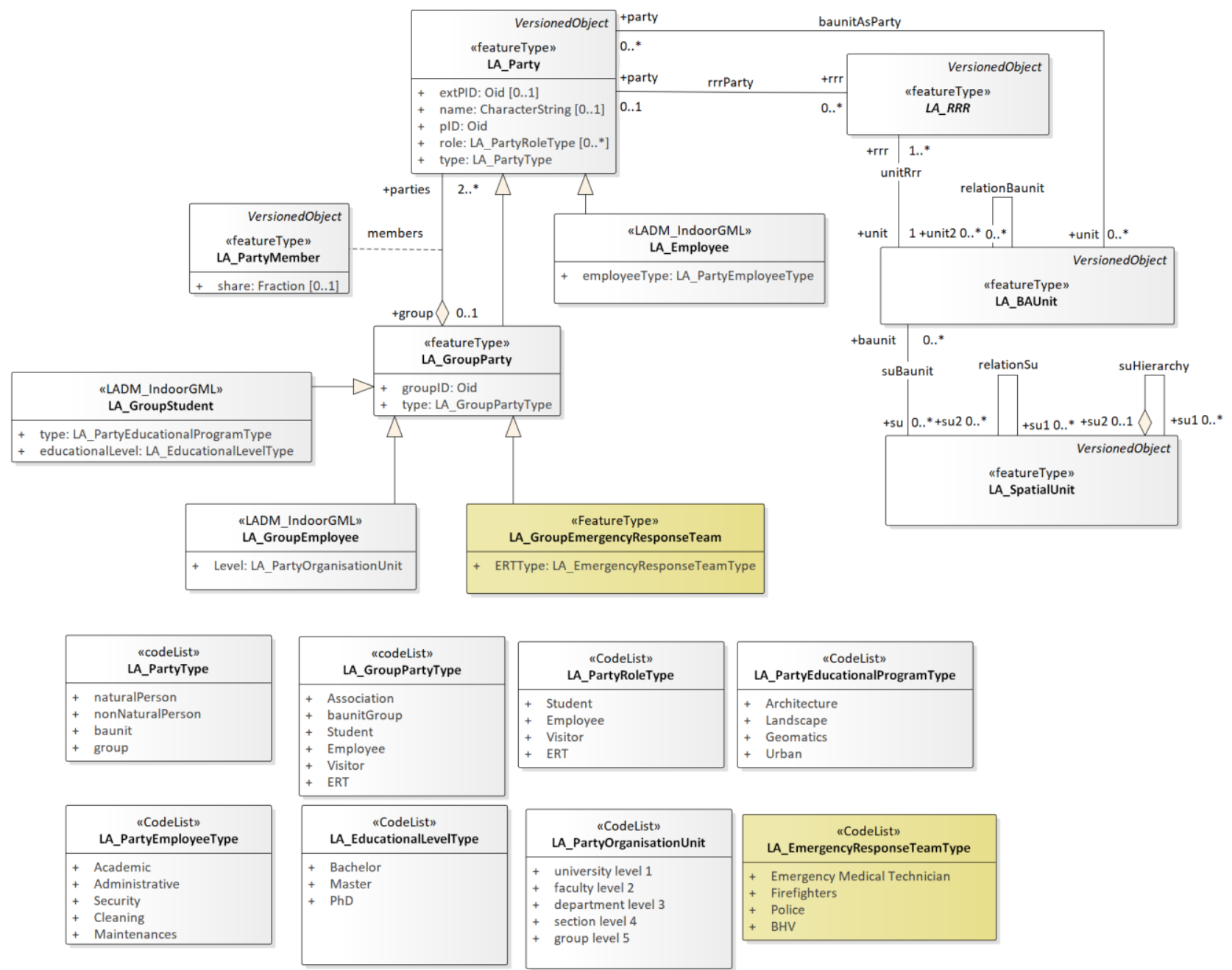

Figure 2. New subclass for the LA_GroupParty class and code list (yellow)

The administrative package consists of two subclasses: the LA RRR and LA_BAUnit. The LA RRR is an abstract class which includes of three subclasses: LA_Right, LA_Restriction, and LA_Responsibilities. "A right is an action, activity or class of actions that a system participant may perform on or use an associated resource (Lemmen et al. 2015)". "A restriction is a state-based or non-state-based entitlement to refrain from doing something (Lemmen et al. 2015)". "A responsibility is a formal or informal obligation to do something (Lemmen et al. 2015)". The LADM standard describes a primary administrative unit ('basic property unit') as an administrative entity, directed to registration (by law), or recordation, consist of zero or more spatial elements ('parcels') against which (one or more) distinctive and compatible RRRs (rights, e.g., ownership rights 
or land use rights, responsibilities, or restrictions) are associated to the whole entity, as included in a land administration system. The LA_RRR class has a timeSpace attribute that responsible for the temporal description of every rights, restrictions, and responsibilities such as every week or every winter as shown in Figure 3.

The timeSpace attribute is different for each user of indoor space based on their RRR. Also, the RRR is changeable based on the condition of the indoor space. For example, the ERT has the access right to enter all indoor spaces during an incident. However, they do not have this kind of access right during the normal condition of the space. Figure 4 shows the same access right for different users of the same indoor space but with different timeSpace conditions. Figure 4a represents students that have private rights to access a lab during the normal office hours, and they do not have access right to the lab out of the normal office hours. In figure $4 \mathrm{~b}$, the cleaning team has private access rights but after the normal office hours. Also, the ERT has the right to access the same space during an incident as shown in Figure 4c.

All of the three type of users had the same rights but the timeSpace attribute has change the temporal condition for their accessibility rights. Users of the indoor space need to find the safest route from their location to the exit area and that is affected by their knowledge of the space. In many cases, the users will try to use the same route that they use every day. However, sometimes this will not be possible based on the incident condition. The users have to discover another route and this may require them to pass through some spaces that are restricted for the users. However, by using the timeSpace attributes of the LA_RRR class, the user can have a conditional access rights based on the condition of the incident. This is allowing more choices for the user to escape from the dangerous zone to safe area.

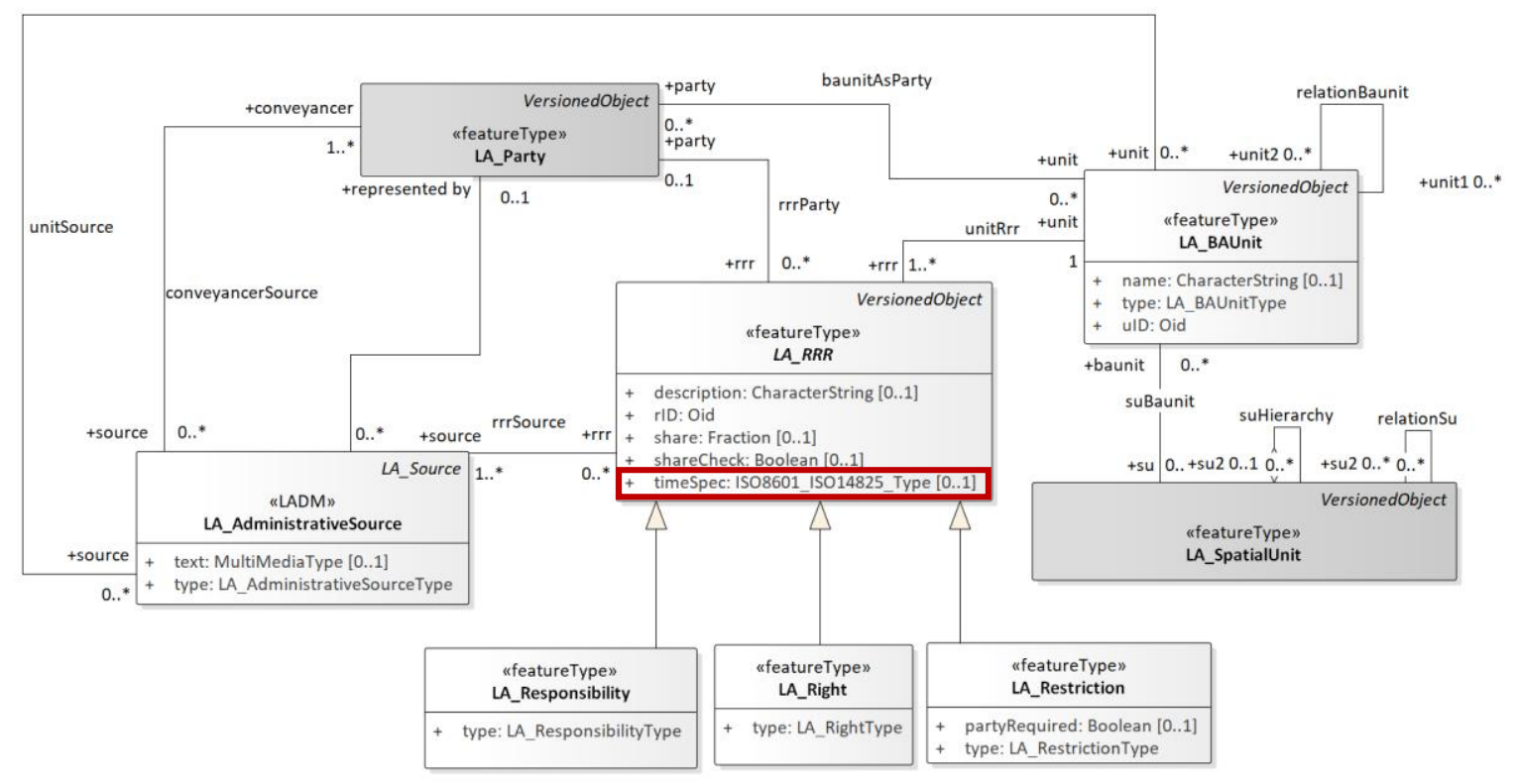

Figure 3. The administrative package of LADM

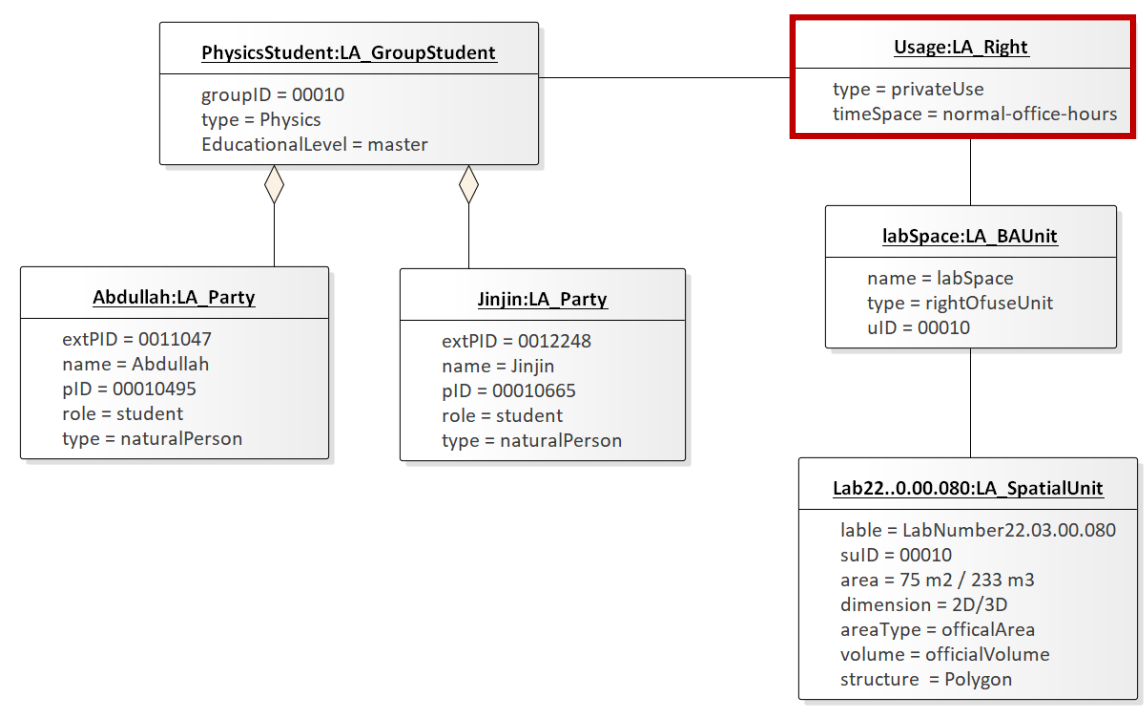



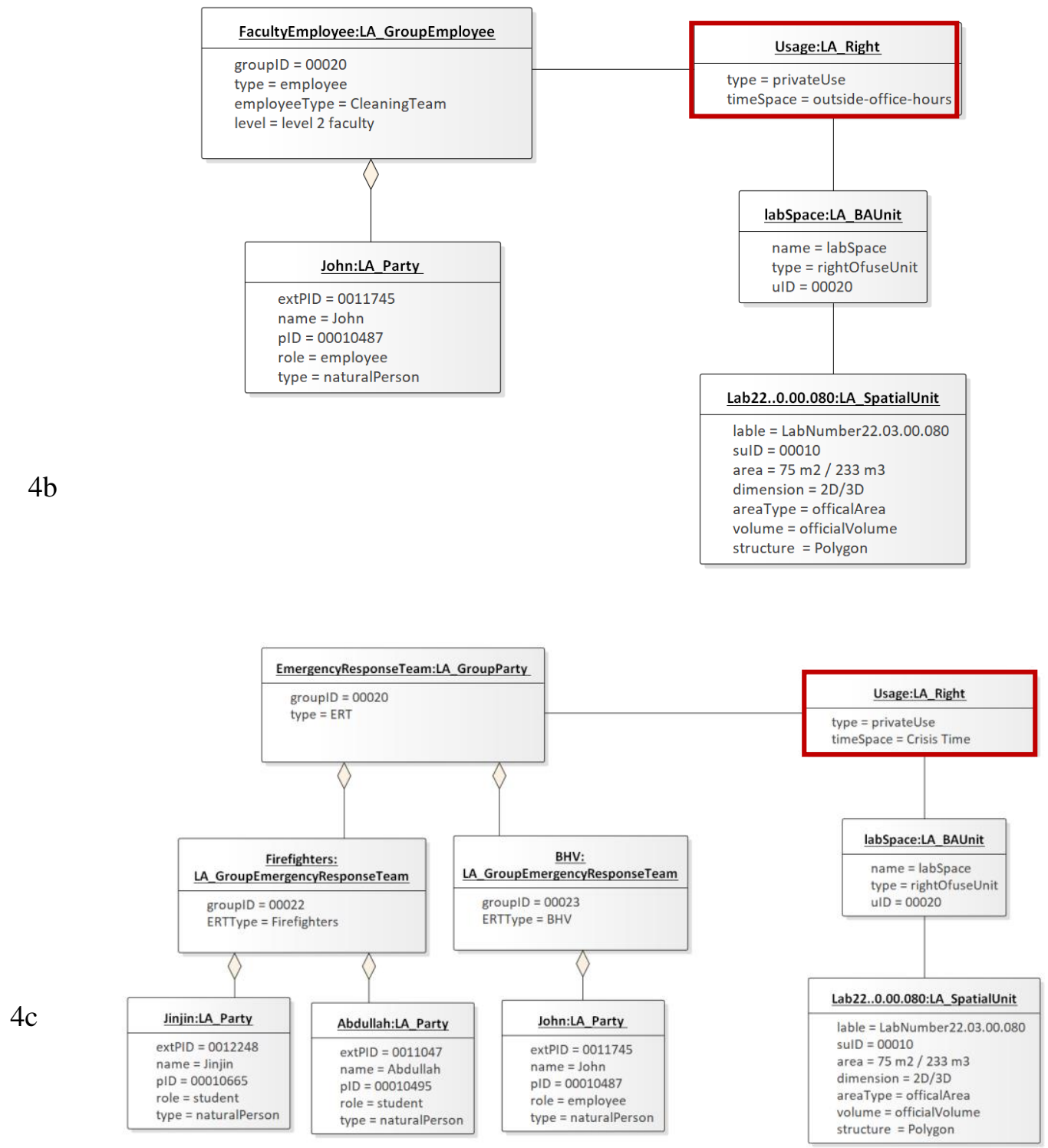

Figure 4. shows different timeSpace conditions for the same access rights

\section{EVACUATION EXERCISE SCENARIOS}

In this section, we investigate the access rights for the users during an incident by preparing two scenarios that are tested in a real exercise in an educational building during normal office time. The evacuation exercise takes place at the Faculty of Applied Sciences at Delft University of Technology, Netherlands. The building of the Faculty consists of six wings that have around 1200 spaces. Seven departments are sharing the building facilities. Therefore, Based on the procedure during an incident, Emergency Response Personnel (ERT) team have to assist users of the building to find their way to the emergency exit. Therefore, we have prepared two scenarios and a 3D model that contains several types of information about each space such as space number, usage, type, specification, organization unit, cleaning frequency (Cleaning), floor finishing (carpet or marble), chair information, department information, and section information as shown in Figure 5. The building consists of the main building front (F wing) and five wings behind (A, B, C, D, and E).
The Physics Engineering building consists of a front block with five wings at the back; this give the building the shape of a hand, looking from above. Each wing has emergency exits at the connection with the front block, in the middle and at the end. The Physics building hosts chemical and physics laboratories, as well as offices, meeting rooms, lecture rooms and technical rooms. The central wing ( $\mathrm{C}$-wing) is the main logistic route from the goods delivery to the front block and its elevators and from there to the rest of the building. A number of laboratories and workshops are located in the $\mathrm{C}$-wing.

\subsection{Scenario A: Bomb reported}

At a certain working day, the Physics Engineering reception is phoned about a bomb being placed in a staircase near the main entrance of the building. The exact location of the bomb is not given. The management decides to evacuate the building, keeping distance from the most eminent danger zones being the staircases of the $\mathrm{C}$-wing and two adjacent wings (B and D), at its connection with the front block. The first responder teams are instructed accordingly and posted on strategic places to 
direct evacuees away from those staircases and out of the building. The slow-whoop is activated, supported by spoken words.

Just when the slow-whoop starts, one of the technicians is in the ground floor corridor of the C-wing transporting a large Dewar vessel with liquid nitrogen. In an attempt to get the Dewar out of the escape route, he misjudges his maneuver, hits an object on the floor and the Dewar topples over. Liquid nitrogen pours out of the Dewar and its vapour starts suppressing breathable air in this segment of the corridor, which is now separated from the rest of the corridor by the closed fire doors. Immediate action is needed to get all people out of this airless section and to block entrance by others. However, a group of 15 workers are now trapped between the bomb area and the airless corridor. To evacuate them, an alternative bypass through a locked workshop had to be opened, leading the group to the safe exit in the middle of the $\mathrm{C}$-wing. Based on the available information about the incident, the dangerous spaces could be highlighted and controlled by the ERT, to assist the users to find an alternative route to the emergency exit, even if the users usually do not have access rights to pass through some spaces. For the first Scenario, we created 2D and 3D evacuation plans to reflect the incident situation and to guide the user to exits of the building as shown in Figure 6. The 2D and 3D plans represent the ground, first and second floors.
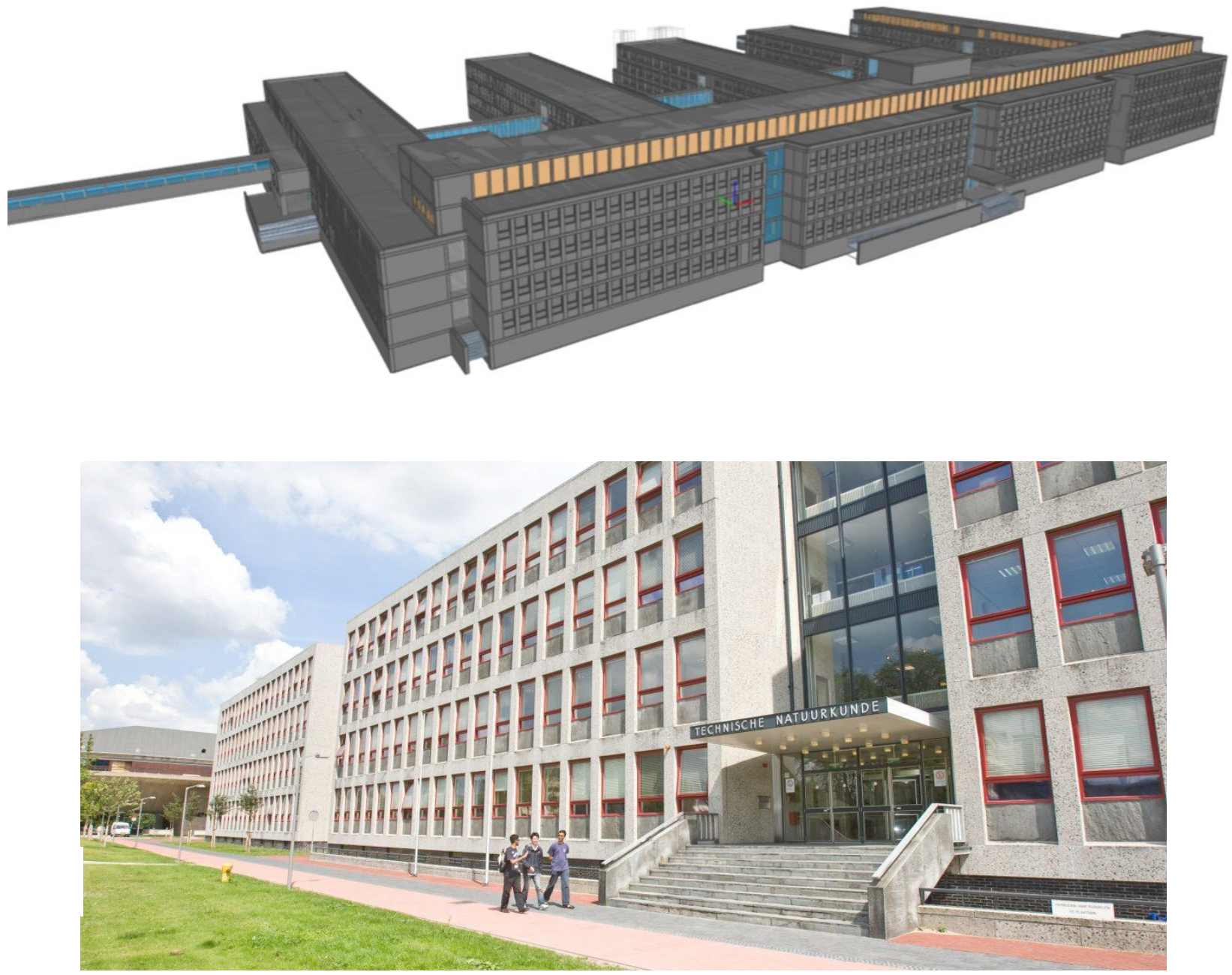

Figure 5. 3D model for Faculty of Applied Sciences (upper) and real picture of the entrance (bottom) (2016) 

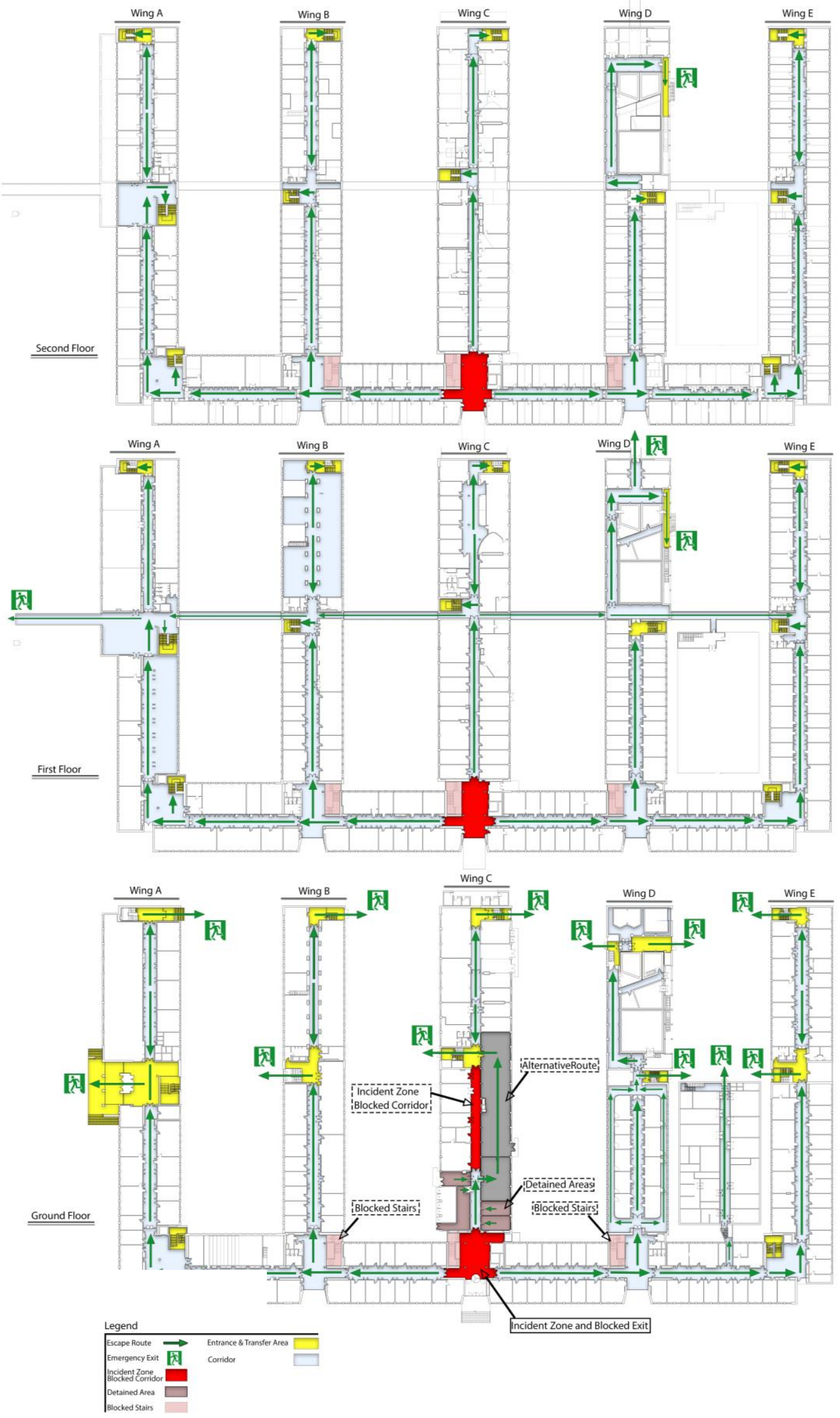


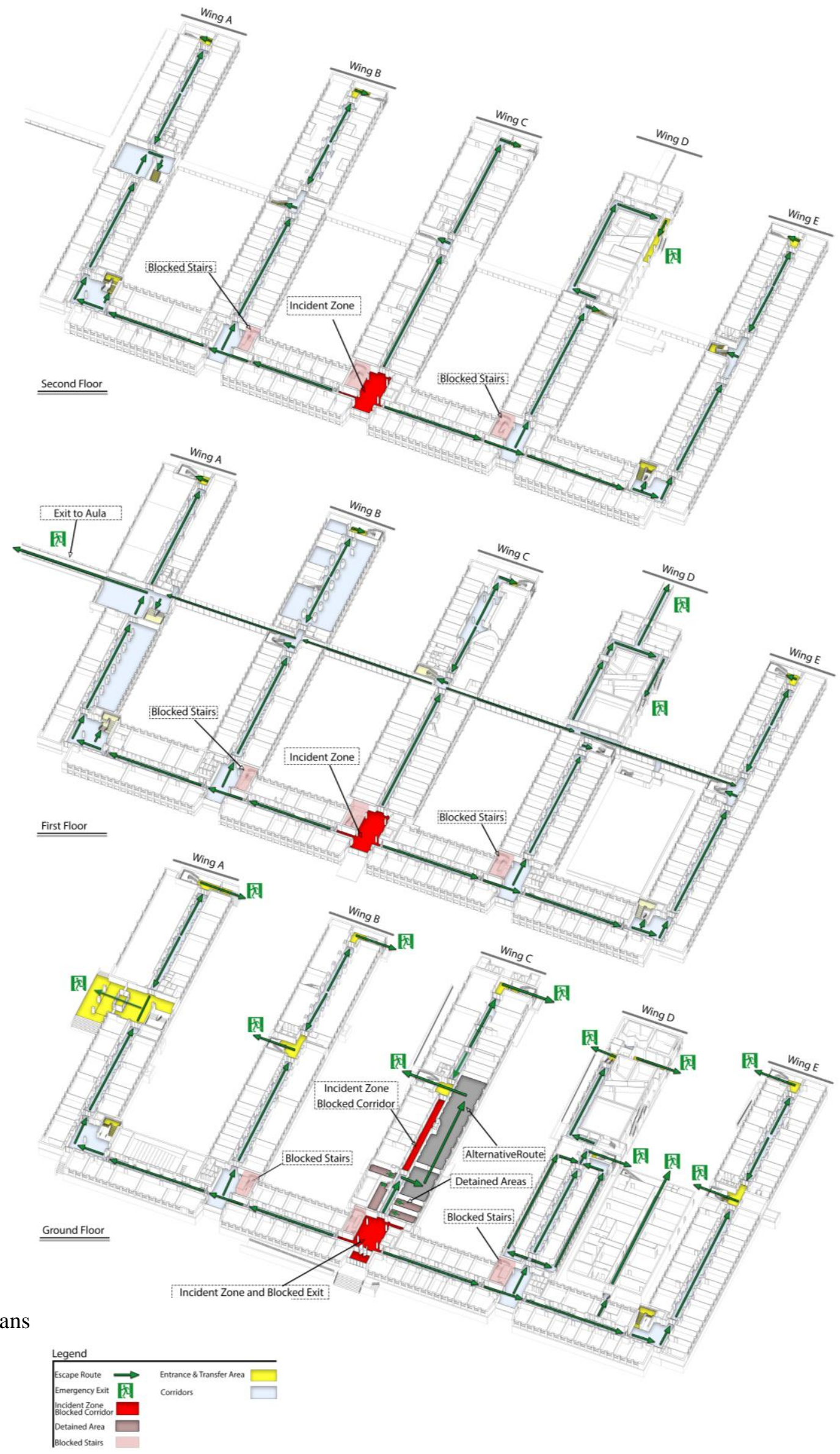




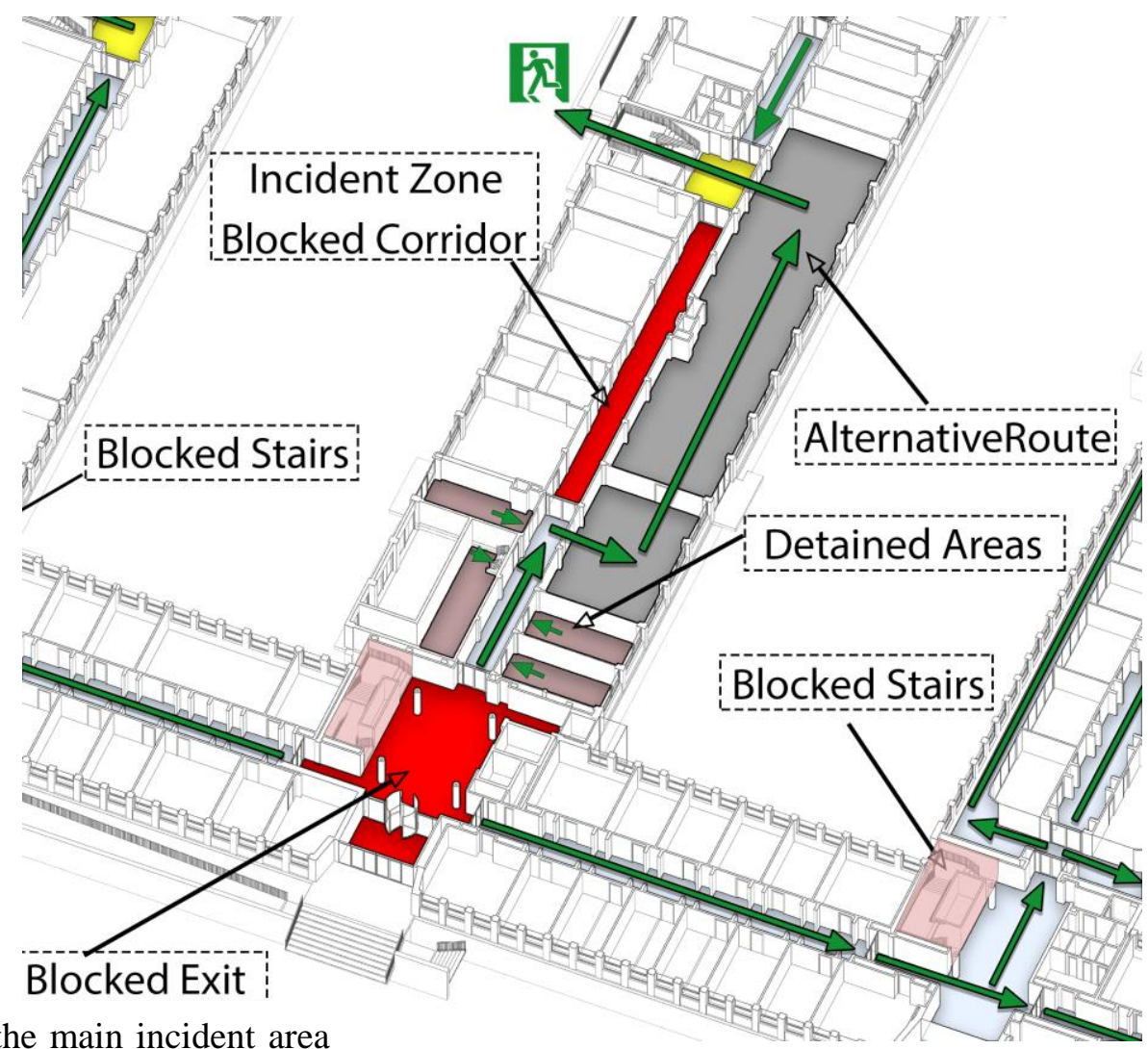

Zoom-in to the main incident area on the ground floor

Figure 6. 2D and 3D plans for Scenario A: Bomb reported

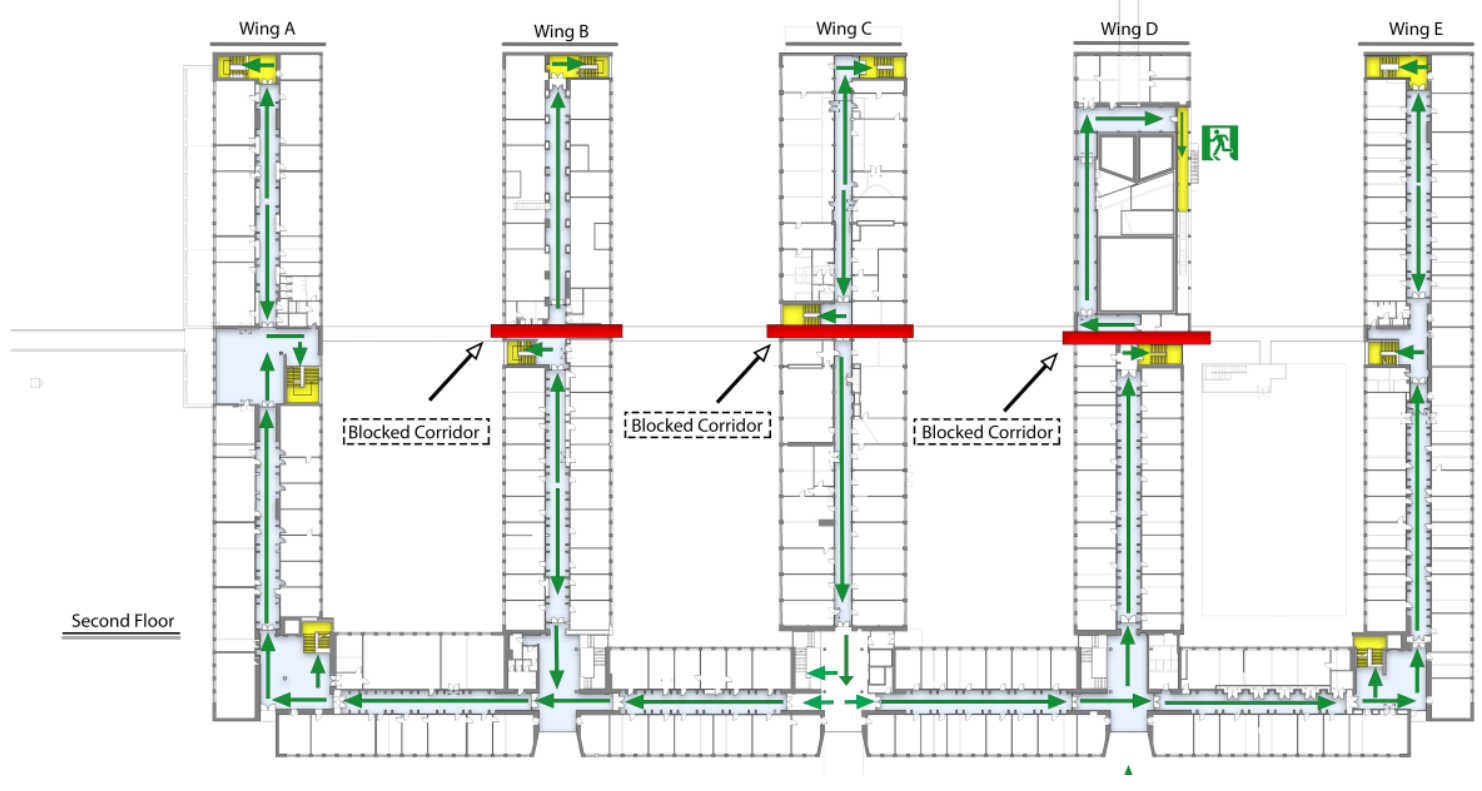



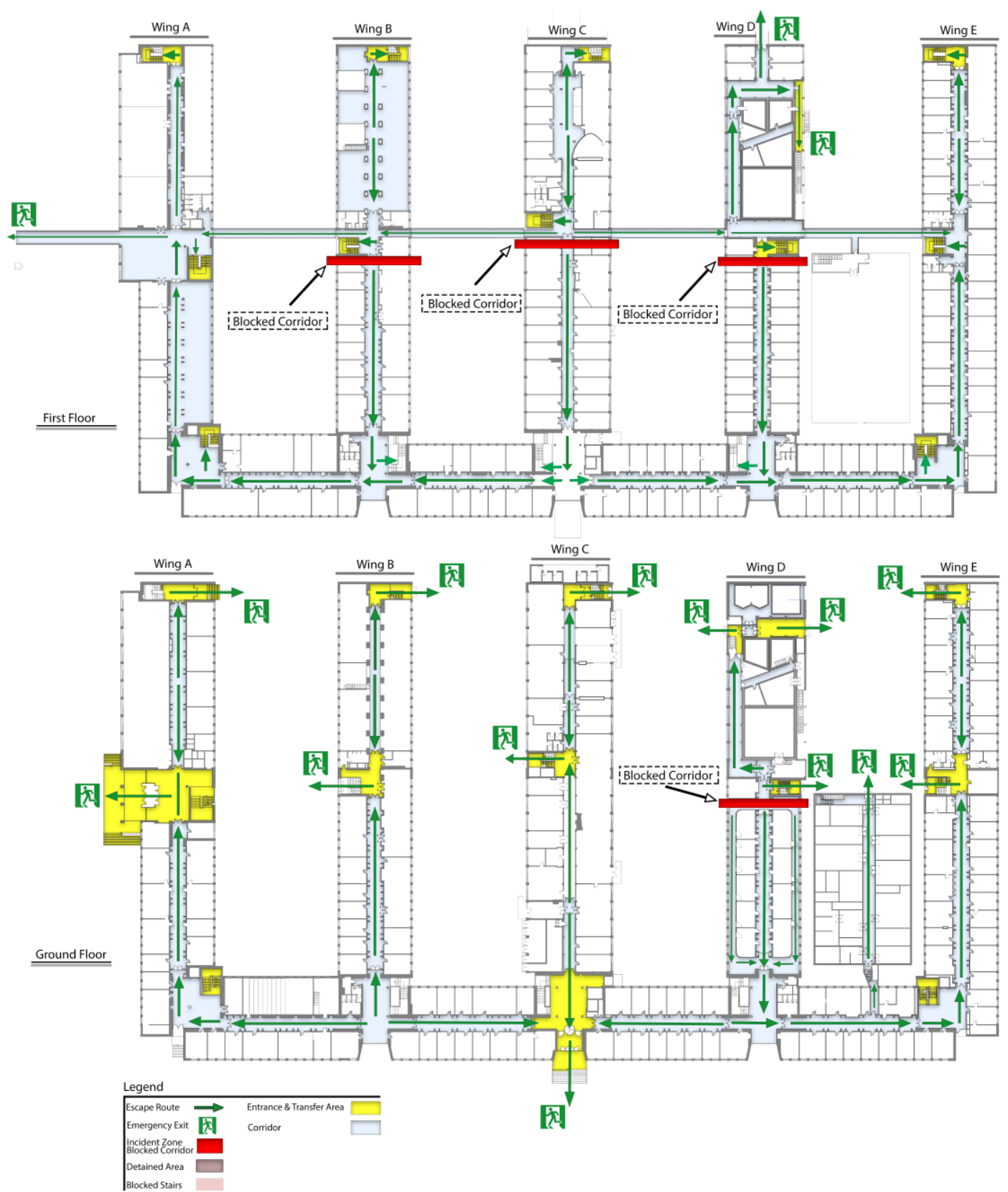

Figure 7. the 2D plan for Scenario B: Flammable gas leakage

\subsection{Scenario B: Flammable gas leakage}

At a certain working day, a leakage of flammable gas from a pipe feeding a laboratory, is noticed in the cellar of front wing near the $\mathrm{C}$-wing. It is unclear how much gas has escaped and how far the gas has spread within the building. Because an explosion risk might be present, the management decides to evacuate the building, keeping distance from the most eminent danger zones. These danger zones are: all floors in the center part of the F-wing and the all floors in the first section of the B, $\mathrm{C}$ and D-wings.

According to the available information about the incident, the ERT team could control the dangerous areas by closing the doors of that space to assist the users to find an alternative route to the emergency exit. The first responder teams are instructed accordingly and are posted halfway the wings to block people from entering the danger zone. The slow-whoop is activated, supported by spoken words. People in the danger zone evacuate quickly through the main entrance (F-wing). People outside of the danger zone are directed to one of the many emergency exits of the A - E wings. Once all people are safely outside, the fire brigade ventilates the building by opening windows while technical staff seals the gas leak. After half an hour, the building is considered safe again. For the Scenario B, 2D evacuation plan has been created to reflect the incident situation and to guide the user to exits of the building as shown in Figure 7. The 2D plan represents the ground, first and second floors.

\section{RESULTS OF THE EVACUATION SCENARIOS}

This section presents the result of the evacuation exercise in the Faculty of Applied Sciences. In the morning of the exercise day, there was a meeting with the Emergency Response Personnel (ERT) team to discuss the ideas behind the Scenarios and to have their approval. They had chosen scenario B for the exercise and they started the preparation for the exercise. There 
were eight observers to cover the exits and to report the user's behaviour during the exercise. When the emergency alarm launched, the users of the building started to move from their offices and labs to the exits of the building. Based on the scenario, the users had to run the opposite direction of the blocked areas. However, most of the users anyhow chose to use the main entrances of the building. Based on the observer's reports, the main entrance of the main building had been used by more than 120 users to escape from the building. The emergency exit that is located in middle wing $\mathrm{C}$ had been used by just 4 users as shown in Figure 8. The middle exit of wing B had been used by 75 users to escape and the other emergency exit of the same wing was closed, and that force the users to change their route to find another emergency exit as shown in Figure 9. The bridge between the Aula building (a neighboring building not show on Figure 5) and the Faculty of Applied Sciences was not used by any user to escape. The second main entrances of the building in wing A had been used by 64 users. The major conclusion of the reports was that users keep following the same route of their daily activity even during an incident.

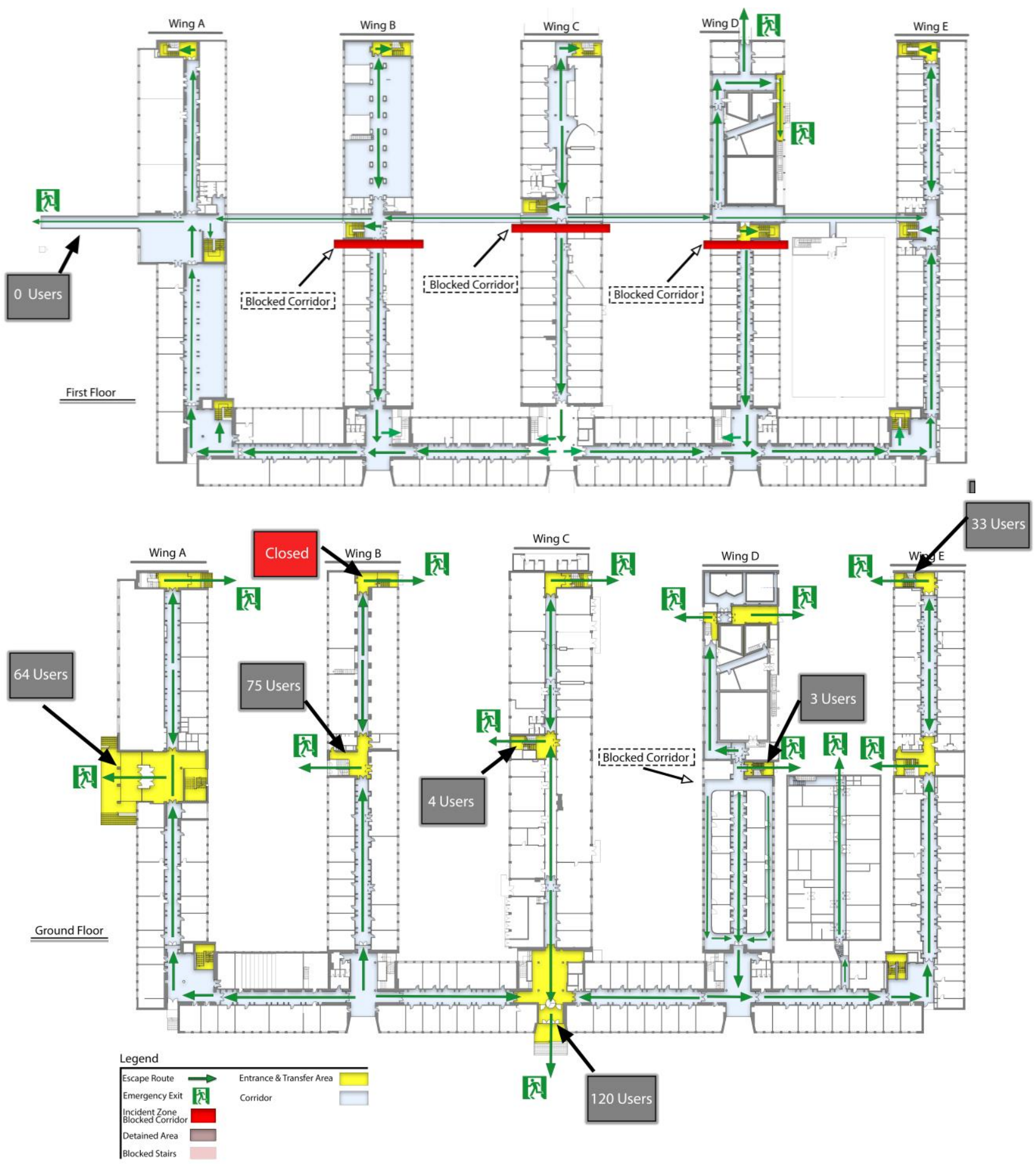

Figure 8. 2D floor plans represents the number of users that escape from the building 


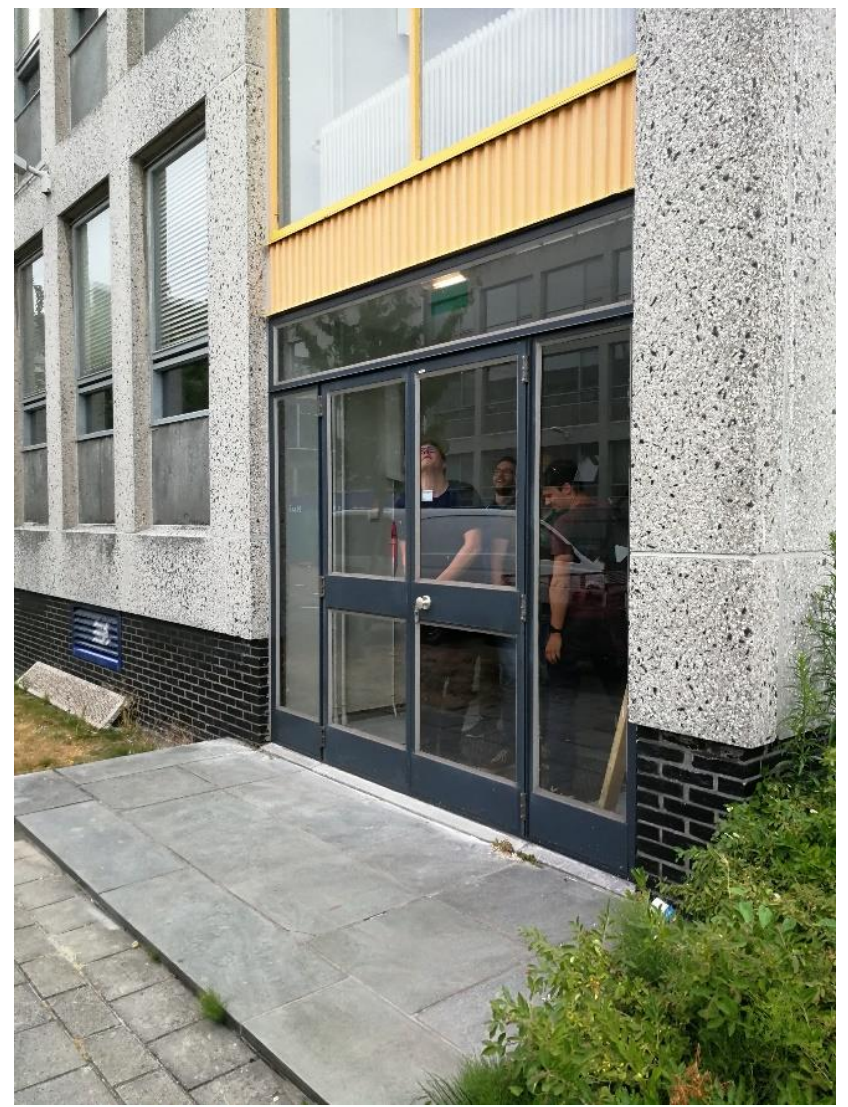

Figure 9. An emergency exits was close during the evacuation exercise

\section{CONCLUSION}

In this paper we extended and tested the conceptual model LADM-IndoorGML during an incident. For this reason, we have prepared two scenarios, to study the behavior of the users during an emergency situation. The second scenario was selected and tested. The initial results have shown that the model can be extended to incorporate information, which can be used during emergency situations to support ER application for ERT or normal users. The most critical component for such situations is the access right and the possibility to change it to be able to guide evacuees through different parts of the building. Evacuation plans according to the new access rights were prepared and provided to the evacuating staff, but they were not used during the evacuation.

The reports of the observers have clearly showed several tendencies, which has to be taken into consideration in future studies:

- The inhabitants of the building kept following the same route that they use during a daily normal activity to get out of the building. Such behavior can be very dangerous and life treating (as in the provided scenario). Further study and developments are needed to estimate which technology or type of guidance would be most convincing and would be accepted by the evacuees. Different approaches should be considered: a personalized navigation sent to a mobile phone, a personalized navigation sent to a prepared user to guide a group of closelocated people, a room or wing-based navigation but then controlled by the ERT people.
- A very interesting observation was also the fact that nobody has chosen to move through a connecting bridge to another building (the Aula). This clearly shows that everyone tried to get out of the building. This raises the attention to the range of the area, which has to be considered in evacuation and the dynamic changes in the indoor/outdoor environments. In this respect pre-pared static evacuation plans may appear to be insufficient. Depending on the treat and the affected area, a completely new routing might need to be provided, which can guide though parts of the building which look 'illogical' to evacuees. In such cases additional sensors or human observations have to be continuously processes as well as the locations of the evacuees.

In this exercise the Wi-Fi data has been recorded but not processed in real time. The data has been stored for further analysis of the user movement during an incident. The future work of this research will expand the current conceptual model of LADM-IndoorGML in order to cover trajectory data related to the cell spaces / spatial units of the indoor model. There are two main types of trajectory data. The first category covers suggested trajectories (so future evacuation routes) according to the user's preferences such as fast path, slow route, shorter route, and longest route. The second category covers the recorded movements of the persons (so current and historic routes). For this purpose, two standards ISO 19141 and ISO 19107 will be considered. ISO 19141 defines a method to describe the geometry of a feature that moves as a rigid body. ISO 19107 covers the feature moves within any domain composed of spatial objects. Based on ISO 19141, the feature may move along a planned route, but it may deviate from the planned route. By using an information model based on international standards (LADM/ISO 19152, OGC IndoorGML, ISO 19141, ISO 19107) we expect that the information will be 
part of the 'building infrastructure' and applications all over the world can understand and use this data when entering or leaving a certain building during both normal and crisis situations. Different types of applications are anticipated to be based on this information model; e.g. mobile indoor routing app, crisis evacuation desktop (or browser-based) application for command center, mobile indoor crisis app (supporting first responders in the field), desktop of server application for analyzing the recorded trajectory data during crisis evacuation (study what did go well, and what did go wrong and should be further improved in the future).

\section{REFERENCES}

Applied Sciences. [Photography]. 2016. Retrieved from http://campusdevelopment.tudelft.nl/wpcontent/uploads/2016/07/TNW_faculteit-1280x600-c-center.jpg

Bonabeau, E., 2002. Agent-based modeling: Methods and techniques for simulating human systems. Proceedings of the National Academy of Sciences, 99, pp.7280-7287.

Hoogendoorn, S. P., \& Bovy, P. H. 2005. Pedestrian travel behavior modeling. Networks and Spatial Economics, 5(2), pp.193-216.

ISO 19152 (E), 2012, Geographic information- Land Administration Model (LADM), ISO 2012, ISO/TC 211 Geographic information/Geomatics, pp. 128.

Lemmen, C., Van Oosterom, P., \& Bennett, R., 2015. The land administration domain model. Land Use Policy, 49, pp.535545 .

Lujak, M., Billhardt, H., Dunkel, J., Fernández, A., Hermoso, R., \& Ossowski, S. 2017. A distributed architecture for realtime evacuation guidance in large smart buildings. Computer Science and Information Systems, 14(1), pp. 257-282.

Pelechano, N., \& Malkawi, A. 2008. Evacuation simulation models: Challenges in modeling high rise building evacuation with cellular automata approaches. Automation in Construction, 17(4), pp. 377-385.

Sime, J., 1984, Escape behavior in fire: 'Panic' or Affiliation?, PhD Thesis, Department of Psychology, University of Surrey, Ann Arbor, 1050, pp.398.

Tang, F., \& Ren, A. 2012. GIS-based 3D evacuation simulation for indoor fire. Building and Environment, 49, pp. 193-202.

Tashakkori, H., Rajabifard, A., \& Kalantari, M. 2015. A new 3D indoor/outdoor spatial model for indoor emergency response facilitation. Building and Environment, 89, pp. 170-182.

Xiong, Q., Zhu, Q., Du, Z., Zhu, X., Zhang, Y., Niu, L., \& Zhou, Y. 2017. A Dynamic Indoor Field Model for Emergency Evacuation Simulation. ISPRS International Journal of GeoInformation, 6(4), pp. 104.

Zlatanova, S., Van Oosterom, P. J. M., Lee, J., Li, K. J., \& Lemmen, C. H. J. 2016. LADM and IndoorGML for Support of Indoor Space Identification. ISPRS Annals of Photogrammetry, Remote Sensing and Spatial Information Sciences, IV2/W1(October): pp. 257-263. 